\title{
Artículo
}

\section{Estudio de Cohorte del impacto y eficacia del proceso de vacunación contra la COVID-19 en la población inmunizada en Costa Rica durante el 2021.}

Cohort study of the impact and effectiveness of the vaccination process against COVID-19 in the immunized population in Costa Rica during 2021.

\section{Autores:}

Dr. Andrés Cairol Barquero ${ }^{1}$, Dra. Wendy Morún Vargas 2, Dr. Luis Felipe Loaiza Sáenz ${ }^{2}$, Dr. Edward Segura Pérez ${ }^{2}$, Dr, Erick Arguedas Hernández ${ }^{2}$, Msc. Sugey González Zuñiga ${ }^{3}$ Sergio Mora Barrantes 4

Coordinador Centro entrenamíento en reanimación, ${ }^{2}$ Centro de entrenamiento en reanimación ${ }^{3}$ Enfermera especialista en Epidemiología ${ }^{4}$ Estudiante Medicina

DOI: https://doi.org/10.54376/rcmui.v4i2.101

Palabras clave: Vacuna contra COVID-19, eficacia, impacto, Costa Rica

\section{Resumen}

Introducción: La nueva enfermedad por coronavirus del subtipo COVID 19 tuvo su aparición en diciembre del 2019 en China. En Costa Rica, el primer caso fue diagnosticado en el 6 de marzo 2020 donde una mujer estadounidense de 49 años de edad aterrizó en un vuelo como turista, el 24 de diciembre del 2020 llegó el primer lote de vacunas de la marca Pfizer ${ }^{\circledR}$ y se inició el proceso de vacunación con personal de salud y adultos mayores. Materiales y métodos: Mediante el análisis de las bases de datos abiertas de casos positivos disponible en el observatorio geográfico en salud mediante el convenio con el ministerio de salud y la base de datos de vacunación publicada en la página web de la CCSS, se revisó del 6 de marzo del 2020 hasta el 11 de noviembre del 2021. Resultados: Se estimó que para la población general con I dosis de vacuna la reducción del riesgo de casos positivos es del 55.4 \% (IC $95 \% 48$ - 63), 
reducción para la hospitalización $69 \%$ ( IC $95 \%$ 37- 85) y la reducción para el fallecimiento del $87 \%$ (IC $95 \% 54-97 \%$ ). Se realizó una prueba de Hipótesis Z, utilizando un valor $\mathrm{p}=0.05$, para un valor critico de 1.96. El valor de $Z$ fue de 23.13, por lo que se rechaza la hipótesis nula, confirmando que si existe un efecto estadísticamente significativo. Conclusiones: el efecto en los casos positivos, hospitalizaciones y fallecimientos de la vacunación contra la COVID-19, es congruente con lo expuesto en otros países con esquemas de vacunación similar, lo que respalda que en el contexto de Costa Rica, el uso de las vacunas mostro un efecto favorable y con evidencia estadísticamente significativa.

\section{Summary}

Introduction: The new coronavirus disease of the COVID 19 subtype had its appearance in in China in December 2019. In Costa Rica, the first case was diagnosed on March $6^{\text {th }}, 2020$, when a 49 -year-old American woman landed on a flight as a tourist, on December $24^{\text {th }}, 2020$, the first batch of Pfizer ${ }^{\circledR}$ brand vaccines arrived, and the vaccination process began with health personnel and elderly adults. Materials and methods: Through the analysis of the open databases of positive cases available in the geographical observatory in health through the agreement with the Ministry of Health and the vaccination database published on the CCSS website, data was revised from March $6^{\text {th }}, 2020$ to November $11^{\text {th }}, 2021$. Results: It was estimated that for the general population with I vaccine dose the reduction in the risk of positive cases is $55.4 \%(95 \% \mathrm{CI} 48-63)$, reduction for hospitalization $69 \%(95 \% \mathrm{CI} 37-85)$ and the reduction of death of $87 \%$ (95\% CI $54-97 \%$ ). A Hypothesis $\mathrm{Z}$ test was performed, using a $p$ value $=0.05$, for a critical value of 1.96. The value of $Z$ was 23.13, so the null hypothesis is rejected, confirming that there is a statistically significant effect. Conclusions: The effect on positive cases, hospitalizations, and deaths of vaccination against COVID-19 is consistent with what has been stated in other countries that had similar vaccination schedules, which supports that in the context of Costa Rica, the use of vaccines showed a favorable effect and with statistically significant evidence.

\section{Introducción}

La nueva enfermedad por coronavirus del subtipo COVID 19 tuvo su aparición en diciembre del 2019 en China, específicamente en la localidad de Wuhan. Los primeros casos llamaron la atención debido a la aparición de pacientes con neumonías graves sin etiología aparente y de avance rápido. El 31 de diciembre del 2019, la Organización Mundial de la Salud (OMS) informó sobre el estado de epidemia en la cuidad Wuhan 
y, al día siguiente, se solicitó el cierre del Mercado donde según las autoridades de China inició la pandemia.

En Costa Rica, el primer caso fue diagnosticado en el 6 de marzo 2020 donde una mujer estadounidense de 49 años de edad aterrizó en un vuelo como turista.

Adicional a caso índice, el esposo y otras 152 personas se convirtieron en sospechosas por lo que fueron tamizadas como contactos del caso positivo. Luego, de manera paulatina, aumentaron los casos de comportamiento no lineal e inició toda la respuesta de la atención de casos aumentaron los casos de comportamiento no lineal e inició toda la respuesta de la atención de casos.

La llegada de las vacunas que se disponen a nivel mundial como alternativa de control de la pandemia, el 24 de diciembre del 2020 llegó el primer lote de vacunas de la marca Pfizer ${ }^{\circledR}$ y se inició el proceso de vacunación con personal de salud y adultos mayores. En Costa Rica se autorizó la utilización de la vacuna de Pfizer-BioNTech ${ }^{\circledR}$ y la vacuna de Oxford/AstraZeneca (AZD1222), debido a que en el mes de Marzo del 2021, solo estas dos vacunas contaban con autorización de emergencia por la FDA.

\section{Materiales y métodos}

Mediante el análisis de las bases de datos abiertas de casos positivos disponible en el observatorio geográfico en salud mediante el convenio con el ministerio de salud y la base de datos de vacunación publicada en la página web de la Caja Costarricense del Seguro Social. Se utilizó Excel ${ }^{\circledR}$ para el análisis de las bases y verificación de variables, JASP ${ }^{\circledR}$, Epidata ${ }^{\circledR}$ y Epi info ${ }^{\circledR}$ para el análisis de datos. Se analizaron los datos mediante medidas de tendencia central variables de dispersión de datos, cálculo de medidas de asociación, coeficiente de correlación. La base de datos se revisó del 6 de marzo del 2020 hasta el 11 de noviembre del 2021 utilizando todas las variables que se publican por parte del Ministerio de salud y con la cobertura de vacunas se utilizó los datos nacionales de I y II dosis publicadas por el ente de salud responsable de la vacunación. Se utilizaron de manera general la tasa de casos nuevos por cada 1000 muestras realizadas, tasa de hospitalización por cada 1000 muestras tomadas y la tasa de fallecimientos por cada 1000 muestras tomadas, para así lograr comparar periodos de tiempo con coberturas altas de vacunación de I dosis y el periodo en donde no se contaba aún con la vacunación, donde el avance de vacunación esta desglosado en la 
fuente original de datos por semana epidemiológico a nivel nacional por lo que no se puede segregar la información por cantones o provincias.

\section{Resultados}

Al revisar los datos de las bases abiertas desde el 6 de marzo del 2020 hasta el 11 de noviembre del 2021 se contabilizaron 561829 casos positivos, 30733 hospitalizaciones y 7125 fallecidos, la tasa de hospitalización es de 54.7 por cada 1000 positivos y la tasa de fallecidos es de 12.68 por cada 1000 positivos. Se calcularon las tasas positivos por cada $100^{\circ}$ muestras realizadas por mes, la diferencia de tasas entre el 2020 y 2021 y la cobertura de I dosis de vacunación contra la COVID-19. Tal como lo muestra el gráfico 1, las tasas de positivos por cada 1000 muestras tomadas es mayor hasta el mes de junio en comparación entre el 2020 y el 2021, desde el mes de junio del 2021 las tasas de positivos en comparación con el mismo periodo del 2020 empezaron a bajar de manera paulatina, donde el mes de julio del 2021 tuvo una reducción de la tasa de 153.1 por cada 1000 muestras, llegando a -191.1 por cada 1000 muestras, resultado de mayor reducción en todo el periodo, esta reducción coincide con el aumento de la vacunación de I dosis ya que al mes de octubre, la cobertura de I dosis fue del $68.3 \%$ de la población con al menos una dosis.

Las tasas por provincia se sacan por cada 1000 personas según la población del Instituto Nacional de estadística y censo, por lo que se calcula la de positivos y fallecimientos. La provincia que muestra una mayor tasa de positivos por cada 1000 personas es San José (115.1 x 1000 personas), seguido de Alajuela (111.6 x 1000 personas), Puntarenas (111,o x 1000 personas), Heredia ( 109.1 x 1000 personas), Limón (98.8 x 1000 personas), Cartago ( 98.7 x 1000) y Guanacaste ( 95.7 x 1000 personas).

Mientras la tasa de fallecimientos muestra a San José con 1.6 x 1000 personas, 3 provincias con 1.4 x 1000 personas: Alajuela, Puntarenas y Heredia, Guanacaste con 1.3 x 1000 personas, Limón 1.1 x 1000 personas y Cartago con 0.8 x 1000 personas, tal como lo muestras las representaciones gráficas en los mapas. 
Mapa por provincia de las tasas de positivos y fallecimientos por cada 1000 personas de personas con COVID-19.
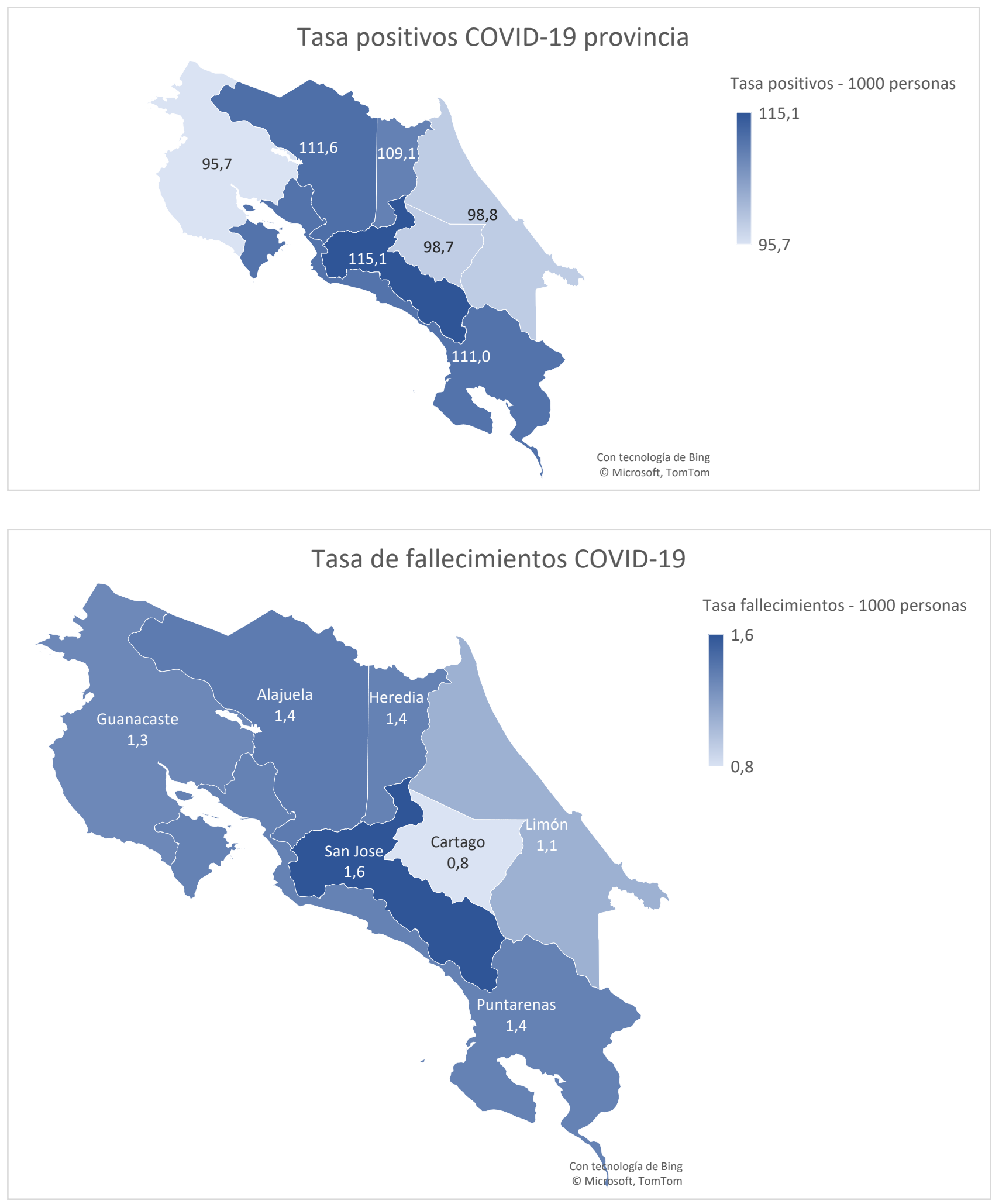
Gráfico 1. Distribución de las tasas de positivos por cada 1000 muestras tomadas, la diferencia de tasas y la cobertura de la I dosis de vacuna contra la COVID-19 por mes, marzo del 2020 a noviembre del 2021.

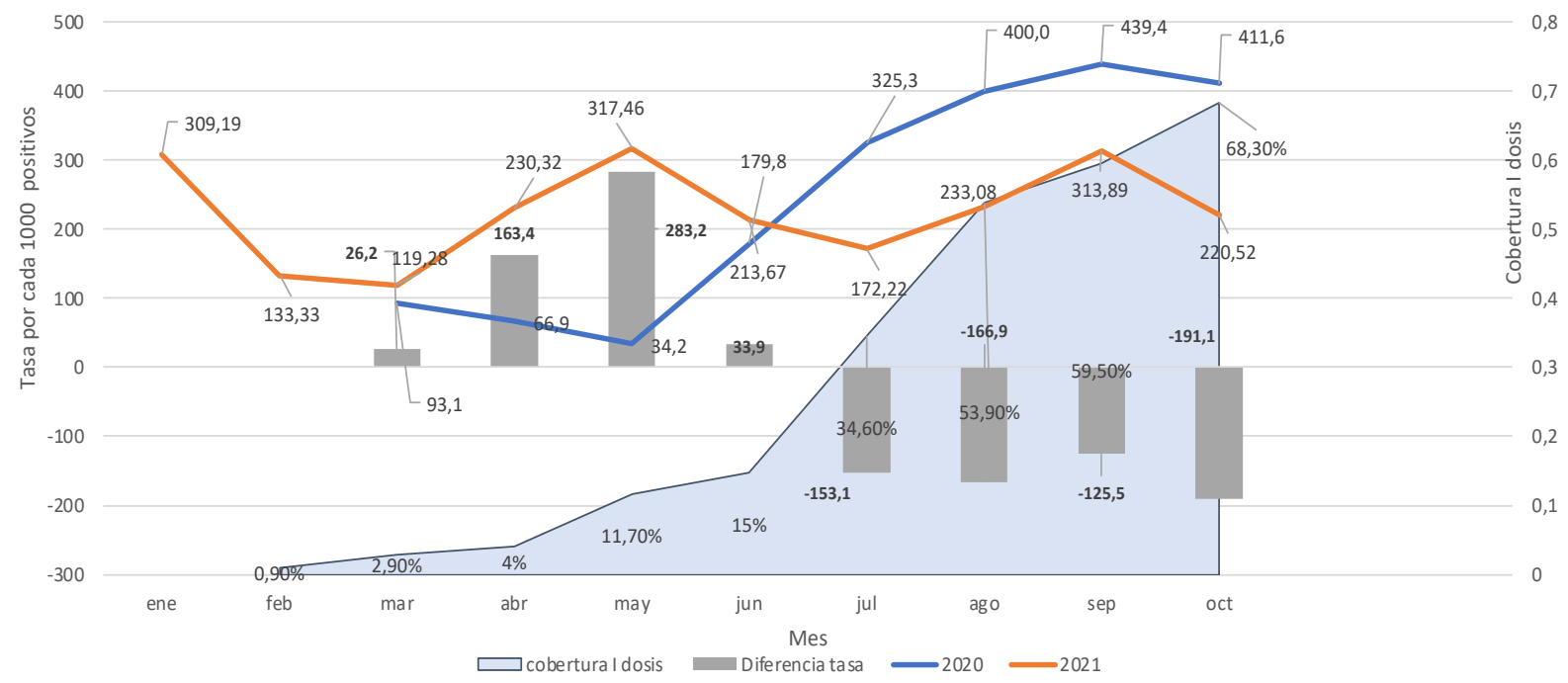

Fuente: elaboración propia

Al revisar las hospitalizaciones encontramos que el comportamiento entre el aumento de la cobertura de I dosis de la vacunación coinciden con la caída de la tasa de hospitalizaciones por cada 1000 muestras, en el mes de julio la tasa se redujo 6.6, agosto 7.1, septiembre 3.8 y octubre 1.9, aspecto que se expone en el gráfico 2. 
Gráfico 2. Distribución de los hospitalizados, tasa de hospitalización por cada 1000 muestras tomadas, la diferencia de tasas mensual y la cobertura de I dosis de la vacunación contra la COVID-19, marzo del 2020 a noviembre del 2021.

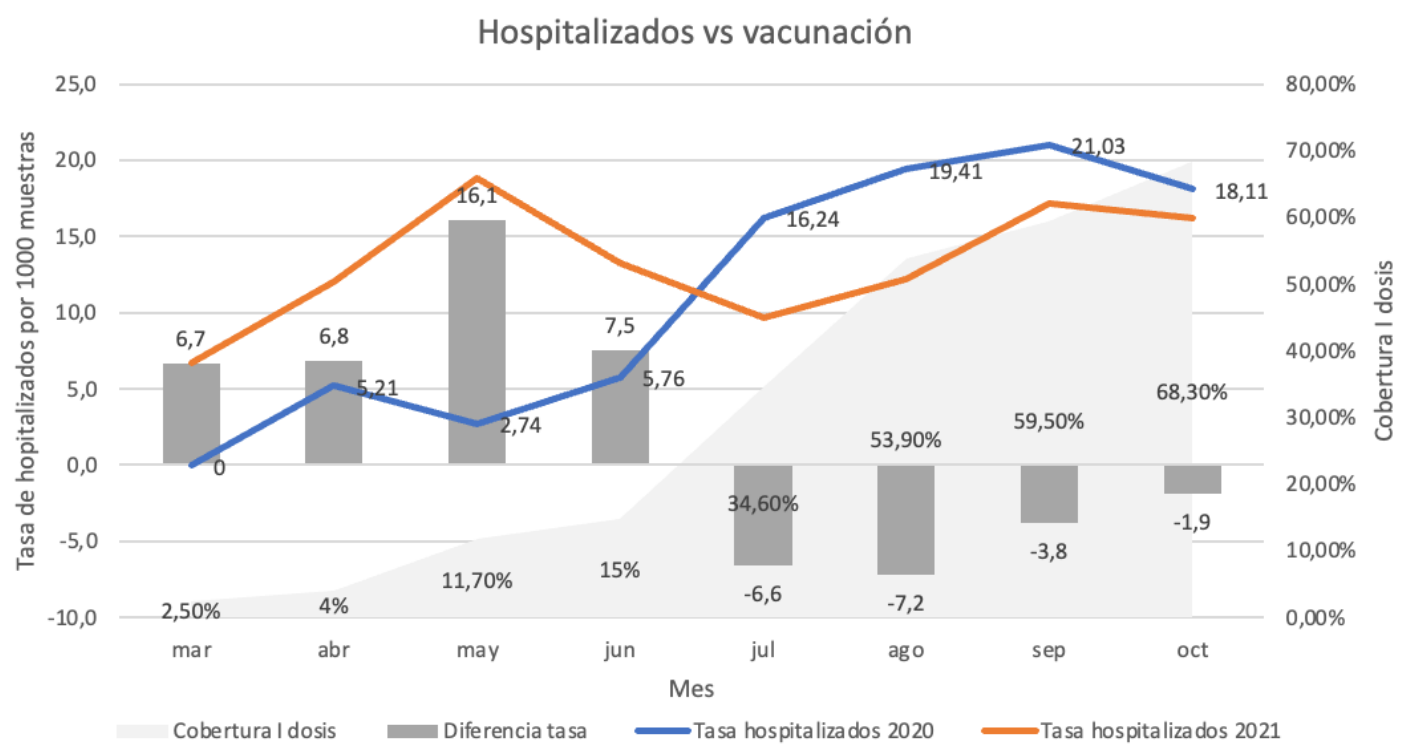

Fuente: elaboración propia.

Los fallecimientos se evaluaron al igual que los positivos y hospitalizados, la tasa de fallecimientos por cada 1000 muestras en el mes de julio del 2020 era de $3.03 \mathrm{y}$ en el 2021 en el mismo mes es de 1.6, como se muestra en el grafico 3, el comportamiento cuando inicia el aumento de la cobertura de I dosis es el mismo que en las otras circunstancias, la tasa de mortalidad llegó a disminuir hasta en 4.02 por cada 1000 muestras. 
Gráfico 3. Distribución de los fallecimientos, tasa de fallecidos por cada 1000 muestras tomadas, la diferencia de tasas mensual y la cobertura de I dosis de la vacunación contra la COVID-19, marzo del 2020 a noviembre del 2021.

\section{Tasa de fallecidos vs vacunación}

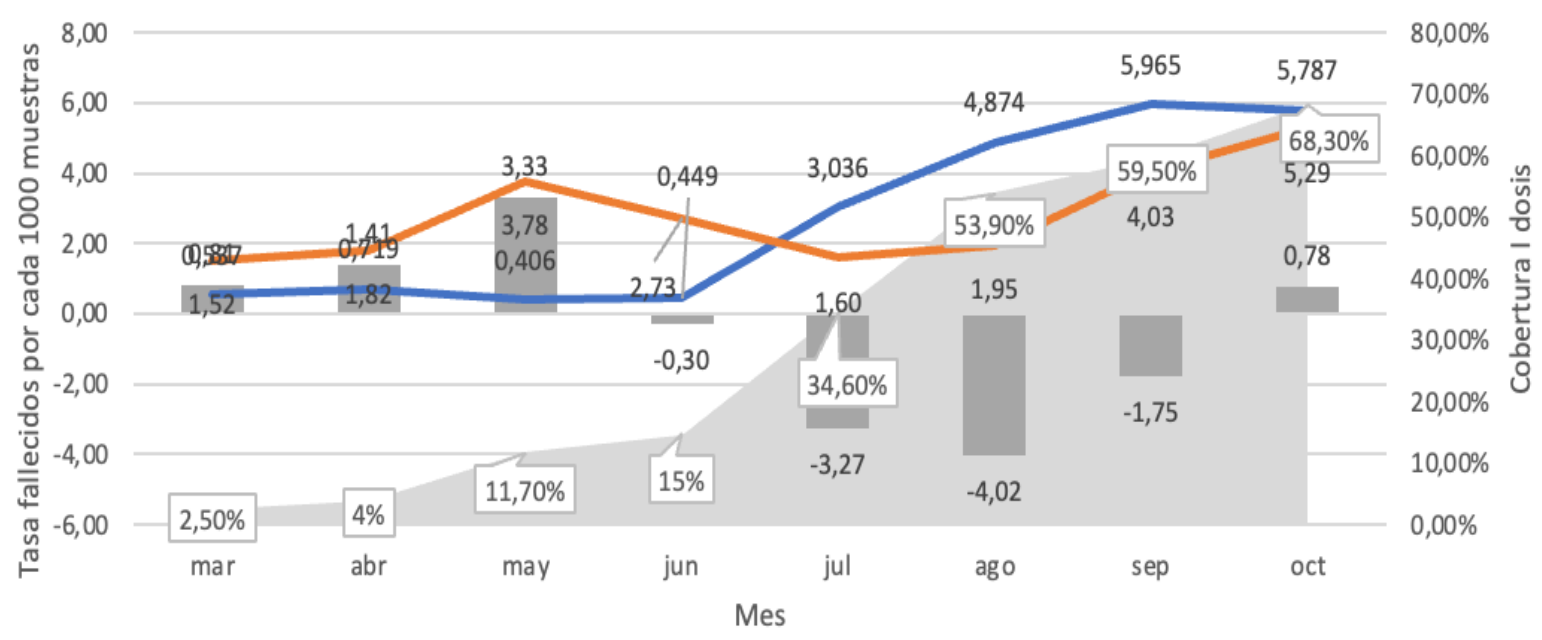

cobertura I dosis - diferencia tasa Tasa de fallecidos $2020 \quad$ Tasa fallecidos 2021

Fuente: elaboración propia.

Se estimó el riesgo relativo durante el periodo de tiempo donde inició la vacunación en la relación con el periodo antes de iniciar la vacunación. La asociación de casos positivos y vacunación mostro un RR 0.44 (IC $95 \%$ 0.37-0.52), para la hospitalización RR 0.31 (IC $95 \%$ o.15 - o.63) y para los fallecimientos RR o.13 (IC $95 \%$ (IC 95 \% o.03o.46), para la población general (tabla 1).

Tabla 1. Efecto de la asociación entre la vacunación y casos positivos, hospitalización y fallecimientos de marzo 2020 hasta noviembre del 2021.

\begin{tabular}{lllll}
\hline $\begin{array}{l}\text { Efecto de la } \\
\text { vacunación }\end{array}$ & RR & IC 95\% & RRR & Valor p \\
\hline Casos positivos & 0.44 & $0.37-0.52$ & $55,4 \%$ & 0.006 \\
Hospitalizados & 0.31 & $0.15-0.63$ & $69,0 \%$ & 0.0013 \\
Fallecimientos & 0.13 & $0.03-0.46$ & $87,0 \%$ & 0.005 \\
\hline
\end{tabular}

RR: riesgo relativo, IC: intervalo de confianza, RRR: reducción del riesgo relativo

Fuente: Elaboración propia. 
Si se evalúa la reducción del riesgo relativo para ser positivo por COVID -19 en la población general es del $55.4 \%$, para la hospitalización es del $69 \%$ y para fallecer por COVID-19 del $87 \%$. Se revisó el comportamiento en el grupo de adulto mayor ya que este grupo se vacunó con prioridad y antes del mes de julio se contaba con una cobertura alta de vacunación de un $95.4 \%$ según datos oficiales de la Caja Costarricense del Seguro Social. Las tasas de personas positivas por cada 1000 muestras positivas bajó drásticamente desde el mes de julio del 2021, donde hay una diferencia de 15.23 por cada 1000 menos y la reducción es sostenida desde el mes de julio del 2021, coincidiendo con el aumento de la cobertura de vacunación general, tal y como lo muestra el gráfico 4 .

Gráfico 4. Distribución de los casos positivos, tasa de positivos por cada 1000 muestras tomadas en e grupo de los adultos mayores, la diferencia de tasas mensual y la cobertura de I dosis de la vacunación contra la COVID-19, marzo del 2020 a noviembre del 2021

\section{COVID adulto mayor}

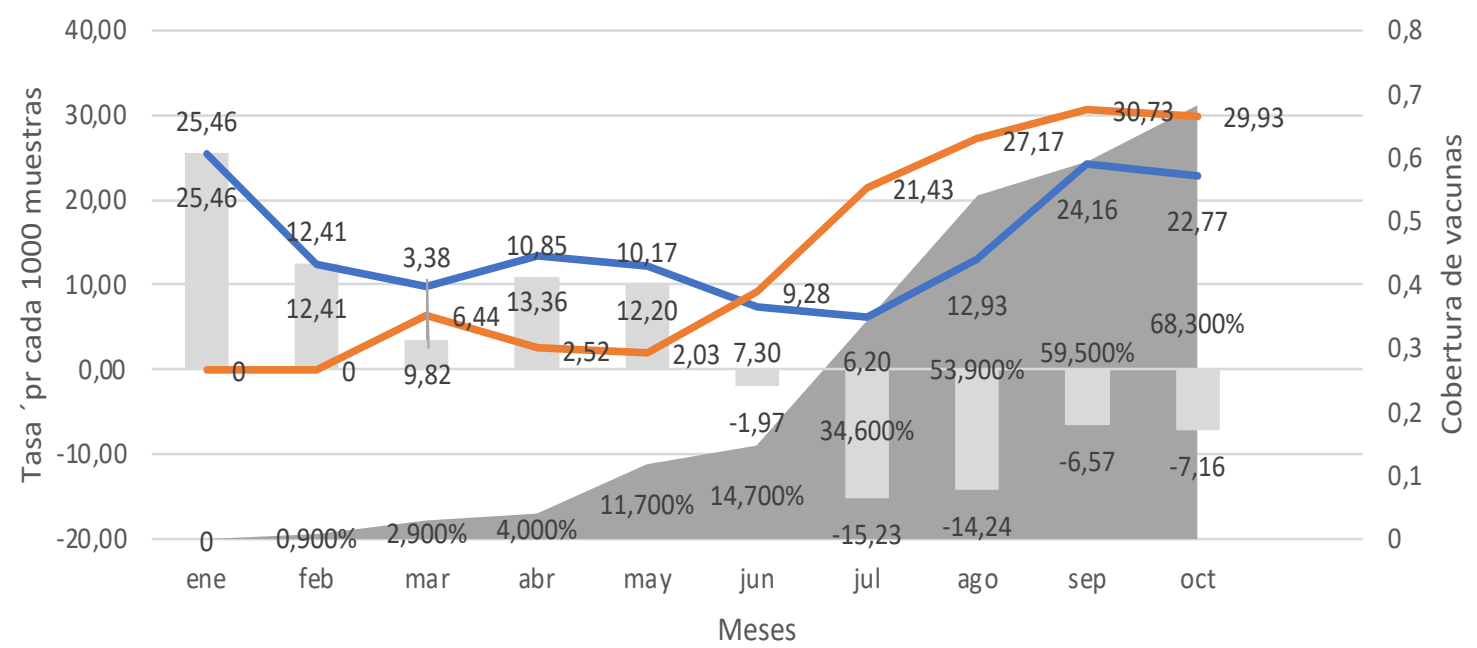

Cobertura vacunas Diferencia tasas 2020

Fuente: elaboración propia. 
La tasa de fallecimientos por cada 1000 muestras tomadas también inició su descenso desde el mes de julio del 2021 y de manera sostenida, lo cual coincide con el aumento de la cobertura de vacunación de I dosis en la población general y ya para ese mes la cobertura de los adultos mayores era superior al $90 \%$, lo cual es consecuente con al reducción en la tasa de positivos, hospitalizados y fallecimientos en este grupo de edad y en la población general (gráfico 5 )

Gráfico 5. Distribución de los fallecimientos, tasa de fallecidos por cada 1000 muestras tomadas en los adultos mayores, la diferencia de tasas mensual y la cobertura de I dosis de la vacunación contra la COVID-19, marzo del 2020 a noviembre del 2021.

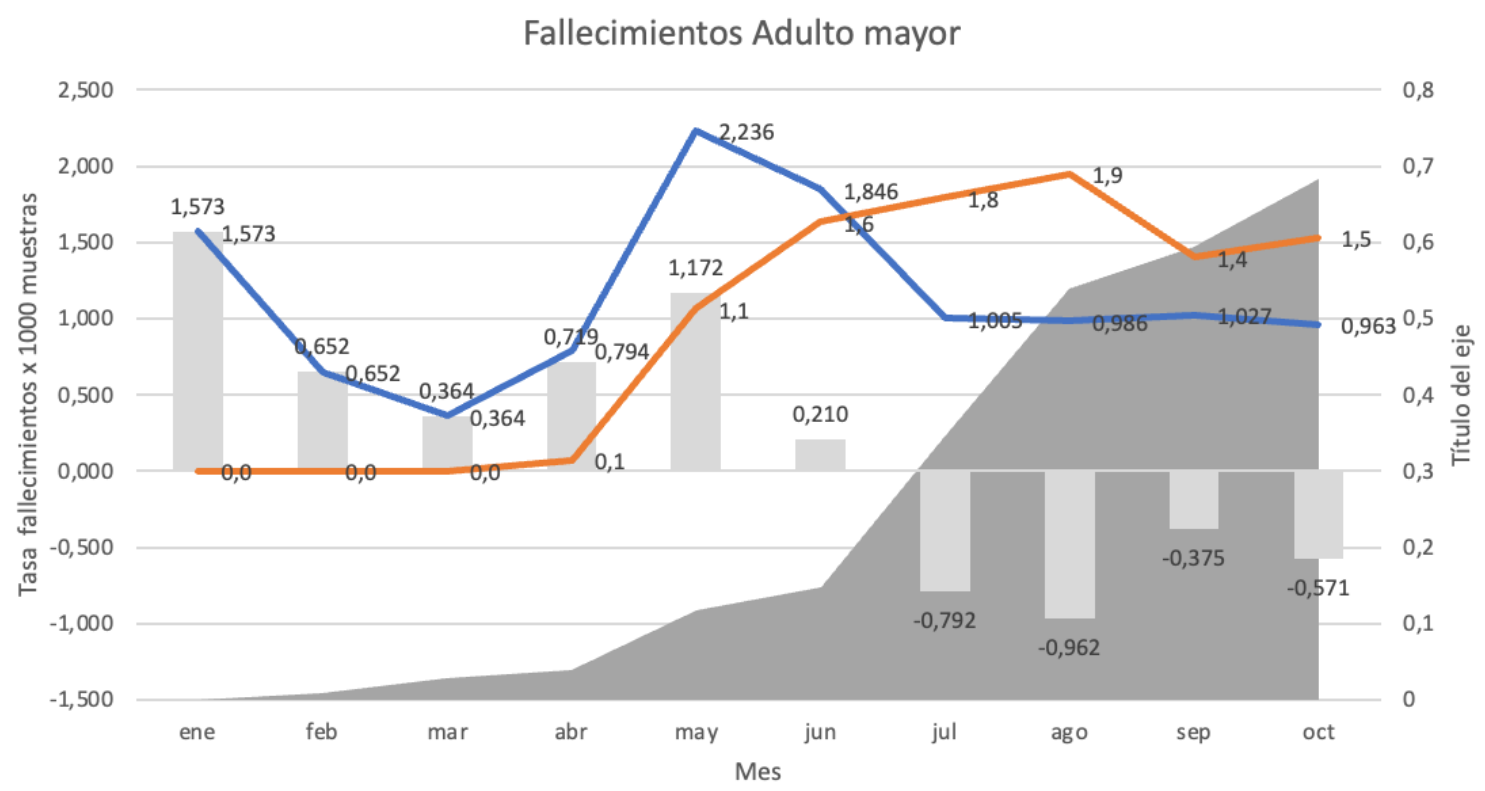

Fuente: elaboración propia.

La relación entre la vacunación y la tasa de positivos y de fallecimientos en el grupo de los adultos mayores mostró que para los casos positivos presentó un RR 0.16 (IC 95 $\% 0.08-0.31$ ) con una reducción del riesgo relativo del $84 \%$. La asociación entre la vacunación y los fallecimientos tuvo un RR 0.47 (IC 95\% 0.43-0.53) y una reducción del riesgo relativo del $53 \%$. 
Si se avalúa la cantidad de casos positivos de los trimestre del año, se nota que cuando aumenta la cobertura de vacunación a un promedio del $50 \%$ durante el III trimestre del 2021, la cantidad de casos disminuye de manera importante. Hay una reducción de 134603 casos durante el IV trimestre del año, lo que coincide con el aumento en la cobertura de vacunación. (gráfico 6)

Gráfico 6. Cantidad de casos positivos de COVID-19 y cobertura de vacunación por trimestre del 2020 al 2021.

\section{Casos positivos vs cobertura vacuanación}

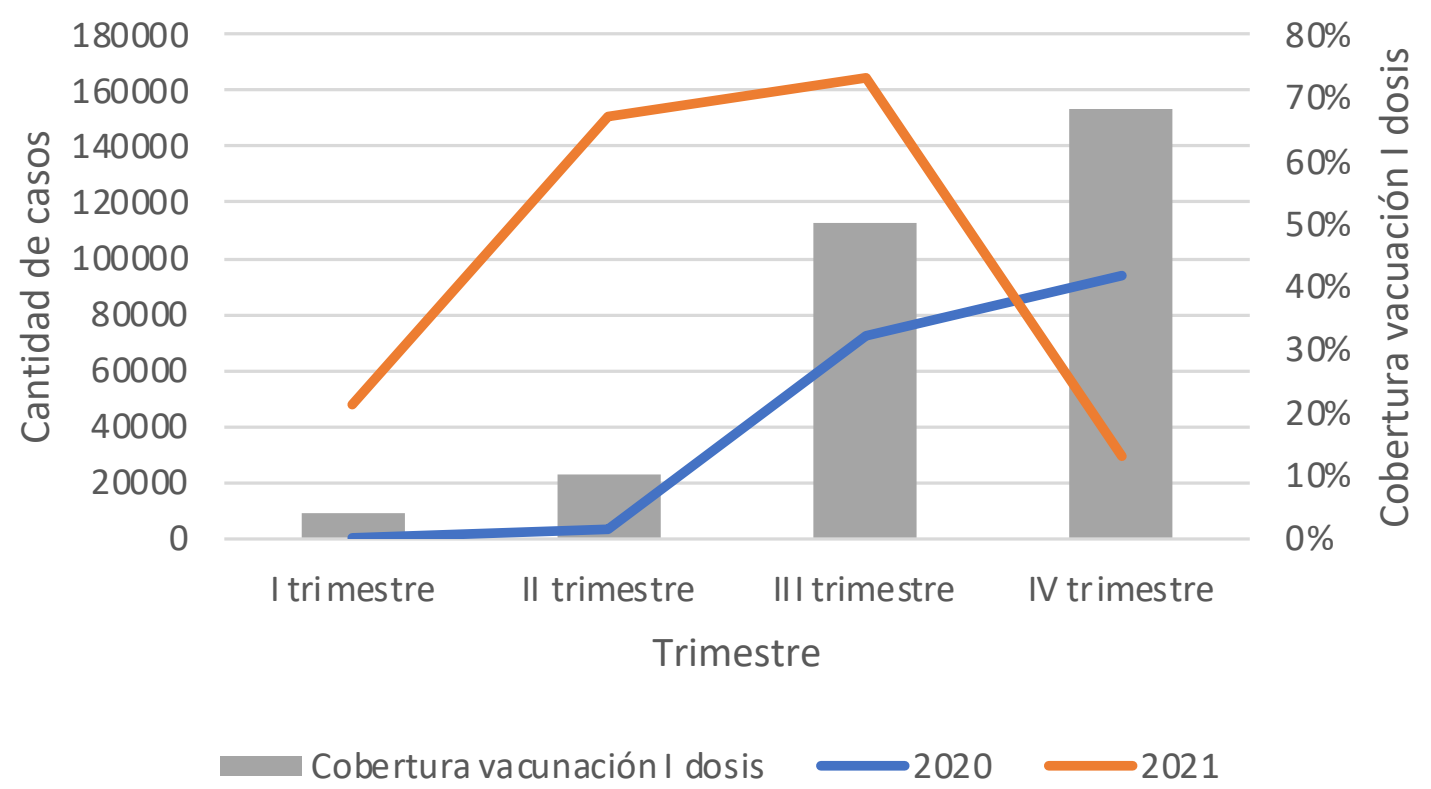

Fuente Elaboración propia.

Si se comparan la cantidad de casos positivos durante el 2021 con el 2020 se nota que la pandemia afectó mucho mas durante el año 2021, donde la media de positivos por día durante el 2020 fue de 51.13 (IC 95\% o - 110.7) y en el 2021 de 760 (IC $95 \% 669.1$ 850.9) sin embargo esta no es una metodología adecuada para comparar dos momentos diferentes en una pandemia que es dinámica y con una tasa de R_t muy variable. La variabilidad de los casos fue muchísimo mayor durante el 2020 que iniciaba la cantidad de casos en el mes de marzo, con un coeficiente de variación del $1.17(117 \%)$ y en el 2021 de 0.12 (12\%). 
Gráfico 7. Cantidad de casos positivos de COVID-19 por año del mes de marzo del 2020 a noviembre del 2021, Costa Rica.

\section{Positivos}

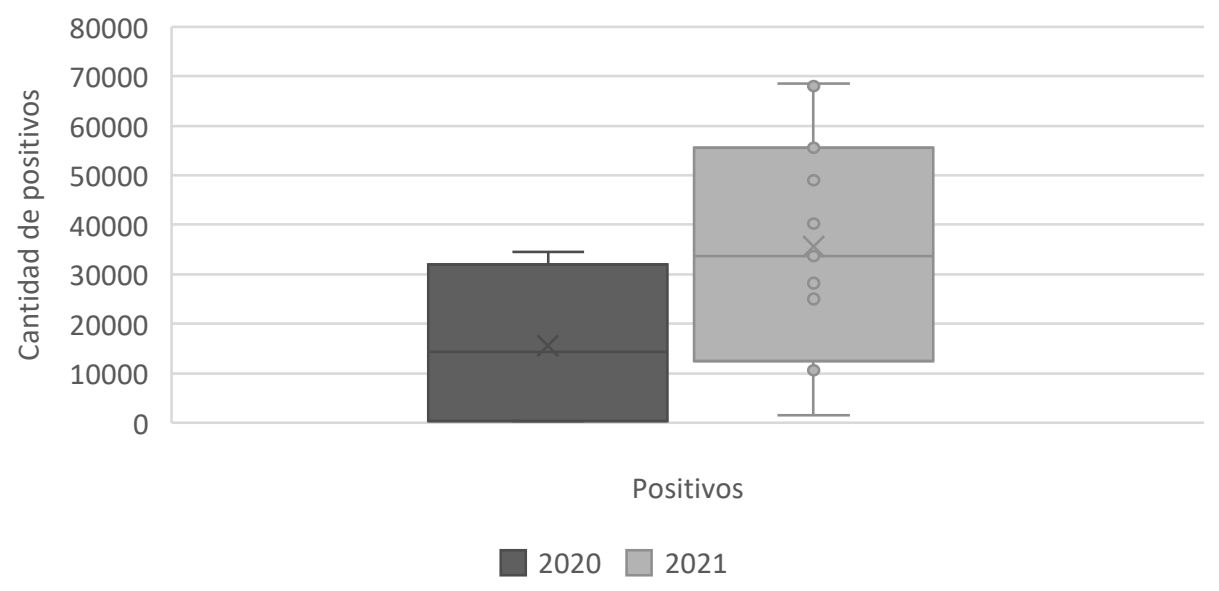

Fuente elaboración propia.

Las tasas sin duda confieren la mejor manera de comparar momentos epidemiológicos diferentes, por lo que se calculó la tasa de positivos por cada 1000 muestras antes de la vacunación masiva y antes de este evento. La tasa de positivos es de 269. 55 por cada 100 muestras positivas en el 2020 y en el 2021 de 226.29 por cada 1000 muestras. (Gráfico 8)

Se comparó a su vez la tasa de positivos por cada 1000 muestras tomadas durante el mes de julio a noviembre de cada año, la tasa durante el 2020 fue de 393.72 por cada 1000 muestras y durante el 2021 fue de 234.92 por cada 1000 muestras, aspecto que se muestra en el grafico 9. Este aspecto muestra que la vacunación si presento un efecto en la reducción de casos positivos si se compara el periodo pandémico pre vacunación masiva y luego de aumentar de manera importante la cobertura de vacunación 
Gráfico 8. Tasa de positivos de COVID-19 por cada 1000 muestras por año del mes de marzo del 2020 a Noviembre del 2021.

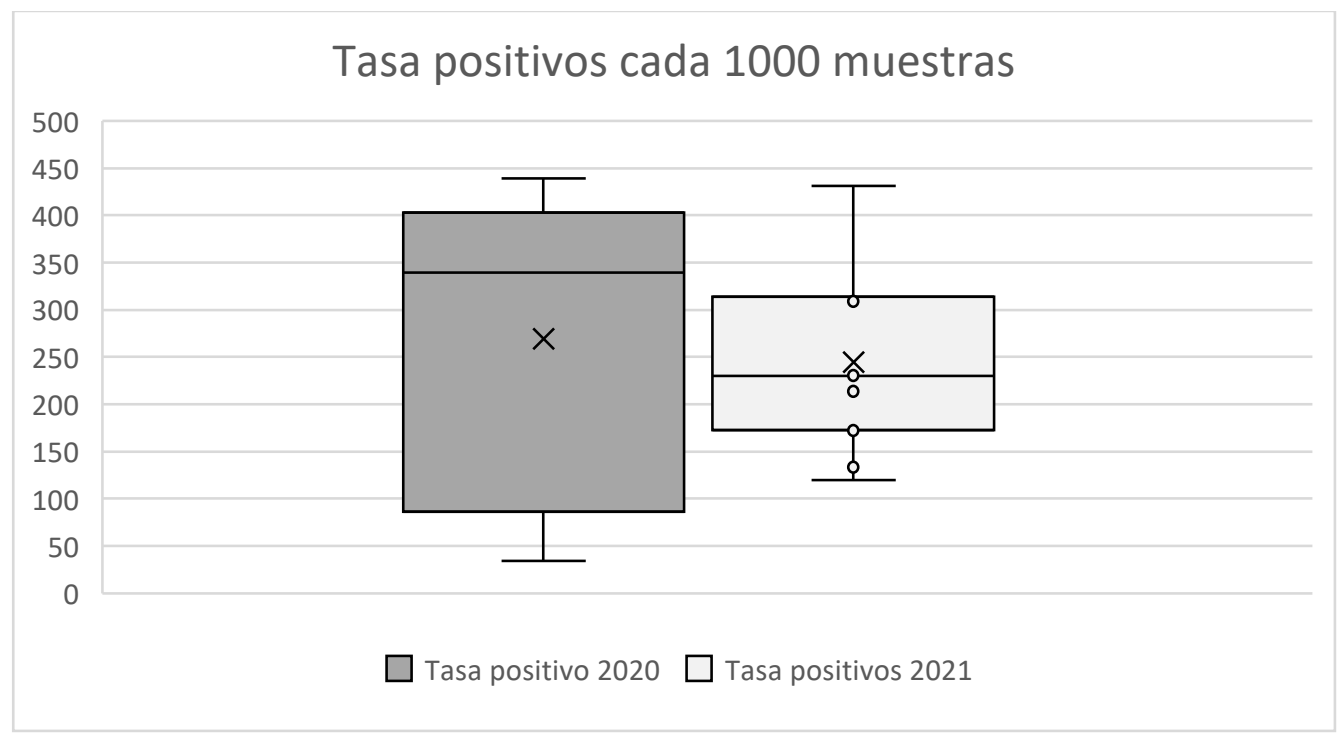

Fuente: elaboración propia.

Gráfico 9. Tasa de positivos de COVID-19 por cada 1000 muestras en el periodo de julio a noviembre del 2020 en comparación con el 2021, Costa Rica.

Tasa positivos $\times 1000$ julio a octubre

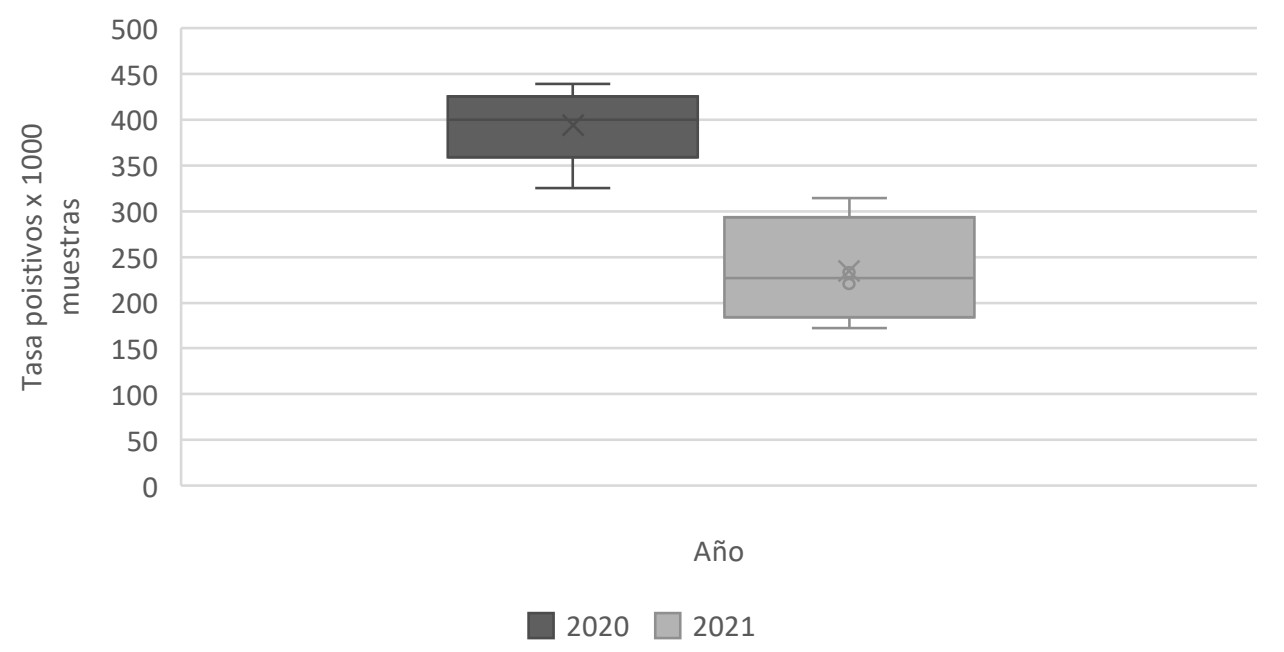

Fuente: elaboración propia. 
Se realizó una prueba de Hipótesis $Z$, donde la hipótesis nula sugiere que la vacunación no afecta sobre la cantidad de casos positivos de COVID-19, por lo que se utilizo una p 0.05, y un valor critico de 1.96 (dos colas) y se compararon los casos positivos del 2020 y 2021. El valor de $\mathrm{Z}$ fue de 23.13, por lo que al ser mayor que el valor critico para el intervalo de confianza, se rechaza la hipótesis nula y se confirma que la vacunación sin tiene un efecto estadísticamente demostrable

\section{Discusión}

En el estudio realizado en el 2020 en 152 sitios de todo el mundo donde se evaluó la eficacia y seguridad de la vacuna de la marca Pfizer, donde la eficacia mostrada con dos dosis de la vacuna es del $94.6 \%$ (IC $95 \% 89.9$ - 97.3). El estudio descrito por Macchia A et al, reporta que luego de revisar los datos en una población de 60 años o más en al cuidad de Buenos Aires, luego de haber utilizado las vacunas de las marcas Aztrazeneca, Sinopharm y Sputnik V con una periodo de 3 semanas entre la primera y segunda dosis, la vacunación se asoció con un $88.1 \%$ (IC $95 \% 96.8-89.2 \%$ ) de la tasa de infección en aquellos que tienen 2 dosis, reducción en la causa de muerte del $65.8 \%$ (IC $95 \% 61.7-69.5 \%$ ). En el estudio realizado por Moghadas SM et al en los Estado Unidos, se describe que en 284 días se logró una cobertura de vacunación del $40 \%$ de la población, a su vez describen que la tasa de ataque se redujo en un $50 \%$ en comparación con los no vacunados. Mientras que la hospitalización se redujo en un 63. $5 \%$ (IC $95 \% 60.3-66.7 \%$ ). McNamara L et al revisaron el panorama de los casos COVID-19 y el comportamiento luego de la vacunación en los Estados Unidos durante el 2020, los resultados mostraron disminuciones relativas del 53\% (IC del 95\%: 50 a 55 ) y del $62 \%$ (IC $95 \% 59$ - 64) entre los adultos de edad 65 a 74 años y 75 años y más, respectivamente, en comparación con los de 50 a 64 años en la cantidad de casos positivos, las admisiones hospitalarias se redujeron en un $39 \%$ (IC $95 \% 29-48$ ) y las muertes por esta causa se redujeron en un $41 \%$ (IC $95 \%$ 14- 69 ).

En el reporte del Ministerio de Salud de Colombia sobre la efectividad de las vacunas contra el COVID-19 en Colombia, realizaron un estudio de Cohorte poblacional donde se incluyeron 3346826 personas y se les dio seguimiento por 4 meses y se utilizó la estimación de la eficacia los modelos de Cox, teniendo como resultado una efectividad para la prevención de la hospitalización del 69.9 \% (IC $95 \% 65.5-73.7 \%$ ), para la reducción de la mortalidad sin hospitalización previa del 74.5 \% (IC $95 \% 71.1-77.5$ $\%)$. Al comparar los resultados obtenidos por otras países tenemos que la reducción en la hospitalización está dentro de los resultados obtenidos la reducción del riesgo en la mortalidad muestra un comportamiento similar a los otros estudios revisados. 


\section{Conclusiones}

Como país consideramos que es necesario que se realicen múltiples estudios no solo epidemiológicos como este, sino también como clínicos donde se evalúe la eficacia de la vacunación contra la COVID-19 que permita medir el efecto sobre la cantidad de casos, hospitalizaciones y fallecimientos relacionados a la pandemia en el contexto específico del país con las circunstancias, coberturas y aspectos que pueden variar los resultados si se comparan con los resultados en otros países. De manera clara, este estudio presenta como principal limitación la imposibilidad del acceso a datos mucho mas específicos sobre centro de atención, factores asociados de las hospitalizaciones y fallecimientos que en el futuro pueden ser de interés científico.

Con los datos analizados de bases de datos de acceso publico tenemos que estadísticamente es demostrable que si existe une efecto positivo sobre la cantidad de casos positivos, hospitalizaciones y fallecimientos secundarios a la COVID-19, donde la cuantificación de la reducción del riesgo relativo para cada evento es positiva y comparable con los resultados a nivel internacional. La caída en la cantidad de casos positivos y por ende hospitalizados y fallecidos coincide con el aumento de la cobertura de vacunación en la población general susceptible y confirma que la vacunación es efectiva en Costa Rica.

\section{Bibliografía}

Macchia A, Ferrante D, Angeleri P, Biscayart C, Mariani J, Esteban S, Tablado MR, de Quirós FGB. Evaluation of a COVID-19 Vaccine Campaign and SARS-CoV-2 Infection and Mortality Among Adults Aged 6o Years And Older in a Middle-Income Country. JAMA Netw Open. 2021 Oct 1;4(10):e213080o. doi: 10.1001/jamanetworkopen.2021.3080o. PMID: 34714342; PMCID: PMC8556631.

Moghadas SM, Vilches TN, Zhang K, Wells CR, Shoukat A, Singer BH, Meyers LA, Neuzil KM, Langley JM, Fitzpatrick MC, Galvani AP. The impact of vaccination on COVID-19 outbreaks in the United States. Clin Infect Dis. 2021 Jan 30:ciabo79. doi: 10.1093/cid/ciabo79. Epub ahead of print. PMID: 33515252; PMCID: PMC7929033.

McNamara L, Wiegand R, Burke R, Sharma A, Sheppard M, Adjemian J et al. Estimating the early impact of the US COVID-19 vaccination programme on COVID19 cases, emergency department visits, hospital admissions, and deaths among adults aged 65 years and older: an ecological analysis of national surveillance data. [Internet]. 
Search.bvsalud.org. 2021 [cited 21 November 2021]. Available from: https://search.bvsalud.org/global-literature-on-novel-coronavirus-2019ncov/resource/zh/covidwho-1506422

Arregocés Castillo L, Fernández Niño J, Rojas-Botero M, Palacios Clavijo A, Galvis Pedraza M, Pinto-Álvarez M et al. [Internet]. Minsalud.gov.co. 2021 [cited 21 November 2021]. Available from: https://www.minsalud.gov.co/sites/rid/Lists/BibliotecaDigital/RIDE/VS/MET/estudi o-efectividad-vacunas-colombia-msps.pdf

Bandyopadhyay A., Chatterjee D., Ghosh K. y Sarkar P. COVID 19: Evaluación epidemiológica y genética del hospedador. Revista Asiática de Ciencias Médicas. 11 (3), 71-76, 2020.

Pal M, Berhanu G, Desalegn C, Kv R. Severe acute respiratory syndrome coronavirus-2 (SARS-CoV-2): An update. Cureus, 2020.

Chan JF-W, Kok K-H, Zhu Z, Chu H, To KKW, Yuan S, et al. Genomic characterization of the 2019 novel human-pathogenic coronavirus isolated from a patient with atypical pneumonia after visiting Wuhan. Emerg Microbes Infect. 9:221-236, 2020.

American Veterinary Medical Association (AVMA). Coronavirus: Detailed taxonomy. Schaumburg, IL: AVMA. Acceso 4 de abril de 2020.

Navarro-Robles E, Martínez-Matsushita L, López-Molina R, Fritz-Hernández J, Flores-Aldana B, Mendoza-Pérez J. Modelo para estimación del comportamiento epidémico de la influenza $\mathrm{A}\left(\mathrm{H}_{1} \mathrm{~N}_{1}\right)$ en México. Revista Panamericana de Salud Pública. 31(4):269-274, 20126.

The factors predicting pneumonia in COVID-19 patients: preliminary results of a university hospital in Turkey. Turkish Journal of Medical Sciences. 20207.

COVID-19 - Respuesta de la OPS/OMS Reporte 23 (31 de agosto de 2020) OPS/OMS | Organización Panamericana de la Salud [Internet]. Paho.org. [cited 11 September 2020].

The factors predicting pneumonia in COVID-19 patients: preliminary results of a university hospital in Turkey. TURKISH JOURNAL OF MEDICAL SCIENCES. 2020. 8Revista Ciencias de la SaludEdición VI • Junio $2021 \cdot$ Vol. IV • Publicación Semestral 